\title{
Putting SMYD3 on the MAP
}

\section{By Lev Osherovich, Senior Writer}

Stanford University and GlaxoSmithKline plc researchers have uncovered the mechanism of action of SMYD3, a histone lysine methyltransferase overexpressed in many lung and pancreatic tumors. ${ }^{1}$ The surprise was that SMYD3 acts in the cytoplasm to regulate the MAPK pathway and not in the nucleus as previously thought. The discovery implies that inhibiting the enzyme can counter activating mutations in MAPK pathway components such as K-Ras.

SMYD3 (SET and MYND domain containing 3) is a member of a family of histone lysine methyltransferases that typically are epigenetic regulators of chromatin

"The beauty and the weakness of hitting SMYD3 is that this is not an essential protein for MAPK signaling. Thus, drugs against SMYD3 are not going to have as many side effects as direct inhibition of the pathway. On the other hand, they may not be as effective as drugs that hit the main components of the pathway."

$$
\text { - Julien Sage, }
$$

Stanford University School of Medicine

Figure 1. SMYD3 in cancer. Mazur et al. have identified the role of SET and MYND domain containing 3 (SMYD3) in K-Ras (KRAS)driven lung and pancreatic cancers.

In certain lung and pancreatic tumors, activating mutations in $K R A S$ or $B R A F(a)$ lead to excessive signaling through downstream MAPK signaling pathways. One branch of the pathway utilizes mitogen-activated protein kinase kinase kinase 2 (MAP3K2) (b) to activate downstream effectors mitogen-activated protein kinase kinase 5 (MAP2K5; MEK5) and MAP kinase 7 (MAPK7; BMK1; ERK-5) (c), which drive tumor growth (d).

The team uncovered that the histone lysine methyltransferase SMYD3 can methylate $\left(\mathrm{CH}_{3}\right)$ MAP3K2 (e), leaving the kinase stuck in an active state to promote hyperactive proliferative signaling. and genetics at the Stanford University School of Medicine. Gozani is an associate professor of biology at Stanford University.

The findings suggest that SMYD3 inhibitors could complement MAPK pathway inhibitors such as GSK's Mekinist trametinib. Mekinist is marketed to treat melanoma with activating mutations in the upstream MAPK pathway regulator BRAF. The drug is in Phase II testing for solid tumors including lung cancers with K-Ras (KRAS)-activating mutations. At least 18 cancer therapeutics targeting the MAPK pathway are in development or on the market (see Table 1, "Selected MAPK pathway compounds in cancer").

"SMYD3 is acting in the cytoplasm to methylate a specific target in the MAPK cascade," said Robert Copeland, EVP and CSO of Epizyme Inc. "The demonstration of a broader role for this enzyme beyond just methylating histones speaks to the interplay between chromatinremodeling factors and other important signaling pathways."

Under a 2011 deal, Epizyme and GSK are discovering compounds against three undisclosed epigenetic targets.

\section{SMYD range}

As part of an ongoing collaboration to characterize the function of

(a) KRAS or BRAF

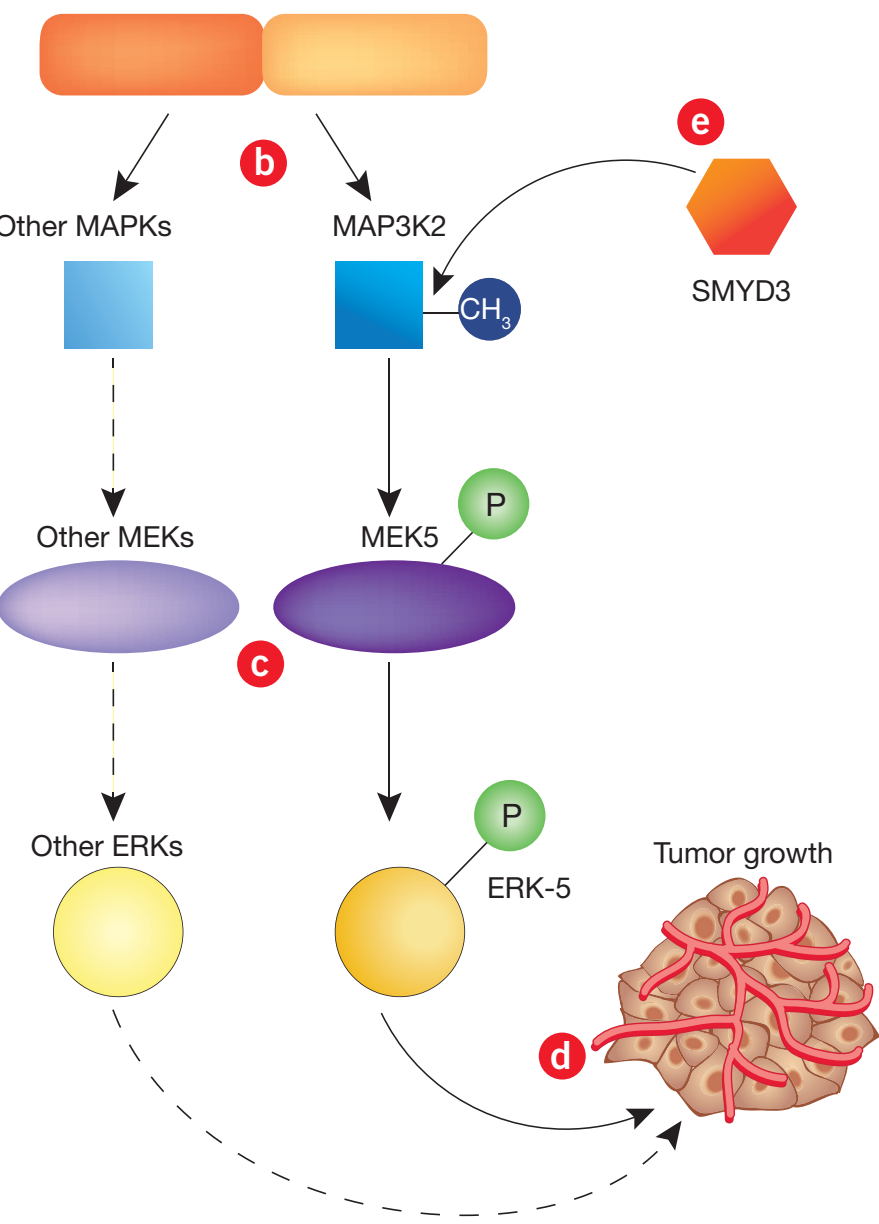


Table 1. Selected MAPK pathway compounds in cancer. Source: BCIQ: BioCentury Online Intelligence

\begin{tabular}{|c|c|c|c|c|}
\hline Company & Compound & Indication & Target & Status \\
\hline Amgen Inc. (NASDAQ:AMGN) & Nexavar sorafenib & $\begin{array}{l}\text { Advanced cancers including colorectal, } \\
\text { liver, breast, renal and ovarian cancers }\end{array}$ & CRAF (RAF1); VEGF receptor & Marketed \\
\hline \multirow[b]{2}{*}{$\begin{array}{l}\text { GlaxoSmithKline plc (LSE:GSK; } \\
\text { NYSE:GSK) }\end{array}$} & Tafinlar dabrafenib & $\begin{array}{l}\text { Melanoma with a BRAF V600E or } \\
\text { V600K mutation }\end{array}$ & BRAF & Marketed \\
\hline & Mekinist trametinib & $\begin{array}{l}\text { Melanoma with a BRAF V600E or } \\
\text { V600K mutation }\end{array}$ & $\begin{array}{l}\text { MAP kinase kinase } 1 \text { (MAP2K1; } \\
\text { MEK1); MEK2 (MAP2K2) }\end{array}$ & Marketed \\
\hline $\begin{array}{l}\text { Roche (SIX:ROG; } \\
\text { OTCQX:RHHBY); Daiichi } \\
\text { Sankyo Co. Ltd. (Tokyo:4568); } \\
\text { Chugai Pharmaceutical Co. Ltd. } \\
\text { (Tokyo:4519) }\end{array}$ & Zelboraf vemurafenib & $\begin{array}{l}\text { Melanoma with a BRAF V600E } \\
\text { mutation }\end{array}$ & BRAF & Marketed \\
\hline Amgen & Nexavar sorafenib & Thyroid cancers & CRAF; VEGF receptor & Approved \\
\hline GlaxoSmithKline & $\begin{array}{l}\text { Tafinlar dabrafenib plus } \\
\text { Mekinist trametinib }\end{array}$ & $\begin{array}{l}\text { Melanoma with a BRAF V600E or } \\
\text { V600K mutation }\end{array}$ & MEK1; MEK2; BRAF & Registration \\
\hline Amgen & Nexavar sorafenib & $\begin{array}{l}\text { Breast, ovarian and liver cancers; } \\
\text { colorectal cancer (CRC) }\end{array}$ & CRAF; VEGF receptor & Phase III \\
\hline $\begin{array}{l}\text { AstraZeneca plc (LSE:AZN; } \\
\text { NYSE:AZN); Array BioPharma } \\
\text { Inc. (NASDAQ:ARRY) }\end{array}$ & Selumetinib (AZD6244) & Non-small cell lung cancer (NSCLC) & MEK1; MEK2 & Phase III \\
\hline $\begin{array}{l}\text { Exelixis Inc. (NASDAQ:EXEL); } \\
\text { Genentech Inc. unit of Roche }\end{array}$ & Cobimetinib (RG7421) & $\begin{array}{l}\text { Melanoma with a BRAF V600E } \\
\text { mutation }\end{array}$ & MEK1; MEK2 & Phase III \\
\hline GlaxoSmithKline & Tafinlar dabrafenib & Solid tumors with BRAF mutation & BRAF & Phase III \\
\hline $\begin{array}{l}\text { Novartis AG (NYSE:NVS; } \\
\text { SIX:NOVN) }\end{array}$ & Encorafinib (LGX818) & Melanoma with BRAF mutations & BRAF & Phase III \\
\hline Novartis; Array BioPharma & Binimetinib (MEK162) & $\begin{array}{l}\text { Melanoma with BRAF mutations; } \\
\text { ovarian cancer }\end{array}$ & MEK1; MEK2 & Phase III \\
\hline $\begin{array}{l}\text { AstraZeneca; Bayer AG } \\
\text { (Xetra:BAYN) }\end{array}$ & Refametinib (BAY 86-9766) & Liver cancer & MEK1; MEK2 & Phase II \\
\hline \multirow[t]{3}{*}{ AstraZeneca; Array BioPharma } & Selumetinib (AZD6244) & $\begin{array}{l}\text { Leukemia; melanoma; thyroid cancer; } \\
\text { breast cancer; CRC }\end{array}$ & MEK1; MEK2 & Phase II \\
\hline & Tafinlar dabrafenib & NSCLC with BRAF mutations & BRAF & Phase II \\
\hline & $\begin{array}{l}\text { Tafinlar dabrafenib plus } \\
\text { Mekinist trametinib }\end{array}$ & CRC & MEK1; MEK2; BRAF & Phase II \\
\hline
\end{tabular}

GlaxoSmithKline

Relapsed or refractory solid tumors

Mekinist trametinib Second-line treatment of NSCLC with

$K$-Ras (KRAS) mutations
Phase II

Pancreatic cancer

\begin{tabular}{|c|c|c|c|c|}
\hline Merck KGaA (Xetra:MRK) & Pimasertib (AS703026) & $\begin{array}{l}\text { Melanoma; pancreatic and ovarian } \\
\text { cancer }\end{array}$ & MEK1; MEK2 & Phase II \\
\hline Novartis & Encorafinib (LGX818) & Solid tumors & BRAF & Phase II \\
\hline $\begin{array}{l}\text { Roche; Daiichi Sankyo; Chugai } \\
\text { Pharmaceutical }\end{array}$ & Zelboraf vemurafenib & $\begin{array}{l}\text { Thyroid cancer with a BRAF V600E } \\
\text { mutation }\end{array}$ & BRAF & Phase II \\
\hline AstraZeneca; Bayer & Refametinib (BAY 86-9766) & Pancreatic and biliary duct cancers & MEK1; MEK2 & Phase I/II \\
\hline BioMed Valley Discoveries Inc. & BVD-523 & Solid tumors & $\begin{array}{l}\text { MAP kinase 1 (MAPK1; ERK-2); } \\
\text { MAPK3 (ERK-1) }\end{array}$ & Phase I/II \\
\hline \multirow{3}{*}{ GlaxoSmithKline } & Tafinlar dabrafenib & Metastatic CRC (mCRC) & BRAF & Phase I/II \\
\hline & \multirow[b]{2}{*}{ Mekinist trametinib } & mCRC & \multirow[b]{2}{*}{ MEK1; MEK2 } & \multirow[b]{2}{*}{ Phase I/II } \\
\hline & & $\begin{array}{l}\text { Solid tumors with BRAF V600 } \\
\text { mutations }\end{array}$ & & \\
\hline \multirow[t]{2}{*}{ Novartis } & Encorafinib (LGX818) & CRC & BRAF & Phase I/II \\
\hline & & & \multicolumn{2}{|c|}{ (Continues on p. 3) } \\
\hline
\end{tabular}


Table 1. Selected MAPK pathway compounds in cancer. (continued)

\begin{tabular}{|c|c|c|c|c|}
\hline Company & Compound & Indication & Target & Status \\
\hline $\begin{array}{l}\text { Teva Pharmaceutical } \\
\text { Industries Ltd. (NYSE:TEVA); } \\
\text { Ambit Biosciences Corp. } \\
\text { (NASDAQ:AMBI) }\end{array}$ & CEP-32496 & Melanoma & BRAF & Phase I/II \\
\hline $\begin{array}{l}\text { Eli Lilly and Co. (NYSE:LLY); } \\
\text { Deciphera Pharmaceuticals LLC }\end{array}$ & LY3009120 (DP-4978) & Melanoma; CRC & BRAF; CRAF; ARAF & Phase I \\
\hline Exelixis; Genentech & Cobimetinib (RG7421) & Solid tumors & MEK1; MEK2 & Phase I \\
\hline Merck; BeiGene Co. Ltd. & BeiGene-283 & $\begin{array}{l}B R A F \text { - or } K R A S \text {-mutation-positive } \\
\text { cancers }\end{array}$ & BRAF & Phase I \\
\hline Pfizer Inc. (NYSE:PFE) & $\begin{array}{l}\text { PD-0325901 plus } \\
\text { PF-05212384 }\end{array}$ & Solid tumors & $\begin{array}{l}\text { MEK1; MEK2; mammalian target of } \\
\text { rapamycin (mTOR; FRAP; RAFT1); } \\
\text { phosphoinositide 3-kinase (PI3K) }\end{array}$ & Phase I \\
\hline \multirow{2}{*}{ Roche; Chugai Pharmaceutical } & RG7304 & Solid tumors & MEK1; CRAF & Phase I \\
\hline & RG7167 & Solid tumors & MEK1; MEK2 & Phase I \\
\hline $\begin{array}{l}\text { Takeda Pharmaceutical Co. Ltd. } \\
\text { (Tokyo:4502) }\end{array}$ & TAK-733 & Solid tumors & MEK1; MEK2 & Phase I \\
\hline
\end{tabular}

epigenetic targets, the Stanford-GSK team started by analyzing gene expression data for 54 histone lysine methyltransferases in a panel of pancreatic cancers with high KRAS activity.

SMYD3 levels were consistently higher in the tumors than in healthy tissue, suggesting that the enzyme might contribute to cancer growth. To test this possibility, the team introduced conditionally activated Kras mutations into a Smyd3 knockout mouse. The animals were less susceptible to pancreatic and lung tumors caused by hyperactive Kras than controls with normal Smyd3. The team observed similar effects with shRNA knockdown of SMYD3 in cultured, KRAS-driven human pancreatic and lung cancer cells and in mouse xenografts.

The next step was to figure out how SMYD3 promotes tumor growth. The team knew that the enzyme methylated something, but identifying the substrates required informed guesswork. One clue came from immunohistochemical experiments in murine pancreatic tumors that showed Smyd3 was predominantly localized in the cytoplasm, not the nucleus.

An in vitro screen for cytoplasmic proteins that could be methylated by SMYD3 pointed to mitogen-activated protein kinase kinase kinase 2 (MAP3K2), a key component of a MAPK pathway branch that is hyperactive in some tumors.

The team went on to show that SMYD3 methylates MAP3K2 in tumor cells, leading to higher MAPK pathway signaling than unmethylated MAP3K2. Surprisingly, the team found that methylation of MAP3K2 by SMYD3 did not directly activate the kinase. Rather, methylation prevented MAP3K2 from being inactivated by a downstream phosphatase called protein phosphatase 2 (PPP2CA; PP2A).

"We expected that the methylation mark would recruit something, but in fact it's the opposite-exclusion of the inhibitor PP2A," said Sage.

Altogether, the findings suggest that SMYD3 renders MAP3K2 constitutively active, leading to broad effects on downstream effector proteins that drive tumor growth (see Figure 1, "SMYD3 in cancer").

Results were reported in Nature and are not patented.

\section{Been there, SMYD that}

SMYD3 thus appears to be a good target for a subset of KRAS-driven tumors. "If you have cancers driven by KRAS and have high levels of SMYD3 or methylation of MAP3K2, these are good candidates for SMYD3 inhibition," said Gozani.

"This study defines a patient population that might benefit from SMYD3 inhibitors," said coauthor Olena Barbash, an oncology R\&D investigator at GSK.

For drug developers, the key question is how targeting SMYD3 compares to hitting MAP3K2 or other targets in the MAPK pathway. "The beauty and the weakness of hitting SMYD3 is that this is not an essential protein for MAPK signaling," said Sage. "Thus, drugs against SMYD3 are not going to have as many side effects as direct inhibition of the pathway. On the other hand, they may not be as effective as drugs that hit the main components of the pathway."

One concern is that SMYD3 could be a player in just one branch of the multipronged MAPK pathway. If so, compensatory changes to other branches of the pathway could render SMYD3 inhibitors ineffective.

However, the Stanford-GSK team showed that SMYD3 knockdown reduced phosphorylation of multiple downstream MAPK targets, suggesting that SMYD3 plays a broad role in amplifying proliferative signaling. Thus, it is possible SMYD3 has other downstream effectors besides MAP3K2.

The principal advantage of

$$
\begin{aligned}
& \text { "If you have cancers driven } \\
& \text { by KRAS and have high levels } \\
& \text { of SMYD3 or methylation } \\
& \text { of MAP3K2, these are good } \\
& \text { candidates for SMYD3 } \\
& \text { inhibition." } \\
& \text {-Or Gozani, Stanford University }
\end{aligned}
$$

may be a better safety profile. Sage said that Smyd3 knockout mice appear to be healthy, so pharmacological blockade of the target is likely to be well tolerated. 


\section{ANALYSIS}

Sage and Gozani think that SMYD3 inhibitors should be paired with compounds such as Mekinist that target core components of MAPK signaling. Indeed, in the Nature study Sage cited evidence that SMYD3 $^{-}$cells were especially sensitive to Mekinist. In vitro and in mouse xenografts, growth of tumor cells lacking SMYD3 was inhibited by a smaller dose of the compound than that needed for wild-type tumor cells.

"We showed deleting SMYD3 could improve the effective dose of MEK inhibitors," said Sage. He noted that drug resistance is more likely to develop when a single drug is given at high doses, so achieving a potent antiproliferative effect with a lower dose of Mekinist "together with SMYD3 inhibitors could reduce the potential for resistance."

The next biology question is whether SMYD3 plays a role in other tumors besides the KRAS-mutant pancreatic and lung cancers examined in the Nature paper. "It will be important to understand whether the modulation of MAPK activity by SMYD3 could be used outside of KRAS-driven tumors," said Barbash. She noted that MAPK pathway abnormalities also occur in some liver cancers.

Osherovich, L. SciBX 7(23); doi:10.1038/scibx.2014.664

Published online June 12, 2014

\section{REFERENCES}

1. Mazur, P.K. et al. Nature; published online May 21, 2014; doi:10.1038/nature13320

Contact: Julien Sage, Stanford University School of Medicine, Stanford, Calif.

e-mail: julsage@stanford.edu

Contact: Or Gozani, Stanford University, Stanford, Calif.

e-mail: ogozani@stanford.edu

2. Cain, C. SciBX 7(19); doi:10.1038/scibx.2014.545

COMPANIES AND INSTITUTIONS MENTIONED

Epizyme Inc. (NASDAQ:EPZM), Cambridge, Mass.

GlaxoSmithKline plc (LSE:GSK; NYSE:GSK), London, U.K. Stanford University, Stanford, Calif.

Stanford University School of Medicine, Stanford, Calif. 